\title{
Contribuições teóricas e abordagens históricas do Efeito Fotoelétrico
}

\author{
Marcos Antônio Barros \\ Professor do Programa de Pós-graduação em Ensino de Ciências e Educação Matemática - UEPB - Doutor em \\ Ensino, Filosofia e História das Ciências - UFBA \\ $\triangle$ marcos_fis@hotmail.com
}

Joana Menara S. Soares

Mestre em Ensino de Ciências e Educação Matemática - UEPB

\begin{abstract}
Resumo:
Uma crescente produção de trabalhos acadêmicos tem analisado a presença de uma abordagem histórica em conteúdos de disciplinas da Física. Contudo, a maioria dos resultados desses estudos não chega aos professores, deixando-os a mercê de histórias (ou estórias) distorcidas e/ou simplificadas, presentes na maioria dos livros didáticos (LDs). Ao considerarmos a possibilidade do uso da História das Ciências (HC) no ensino e a importância de se ensinar um conteúdo relacionado a uma Física dita moderna, torna-se relevante examinar como se dá essa articulação nos livros didáticos, uma vez que esses são a principal ferramenta didática a que o docente tem acesso. A pseudo-história encontrada em alguns LDs de Física reforça conhecidos mitos científicos e transmitem falsas concepções históricas a estudantes e professores. Tais percepções evidenciam a necessidade de trabalhos que realcem abordagens históricas de caráter válido acerca de conteúdos de Física. Assim, considerando a importância da HC, destacamos a relevância de se examinar a presença dessa inter-relação nos LDs de Física para o ensino superior, investigando como o conteúdo histórico referente ao efeito fotoelétrico está apresentado nesses materiais. Para isso, tomamos como base dois critérios: o primeiro se refere à fidelidade do material analisado, em comparação com os trabalhos originais, levando em consideração a abordagem histórica do efeito fotoelétrico e o segundo critério consiste na análise da presença de concepções inadequadas em materiais didáticos do ensino superior sobre aspectos históricos da ciência, como mitos, inadequações e anacronismos.

Palavras-chave: Efeito fotoelétrico; História das Ciências; Livros Didáticos; Física Moderna.
\end{abstract}

\section{Theoretical contributions and historical approaches of the Photoelectric Effect}

\begin{abstract}
:
A increasing production of academic papers have analyzed the presence of a historical approach in contents of disciplines of physics. However, most of the results of these studies do not reach the teachers, leaving them at the mercy of distorted and / or simplified stories, found in most textbooks (TB). When considering the possibility of using the History of Science (HS) in education and the importance of teaching a content related to a so-called modern physics, it is relevant to examine how this articulation appears in the textbooks, since these are the main teaching tool that the teacher has access. The pseudo-history found in some TB of Physics reinforce known scientific myths and convey false historical concepts to students and teachers. Such perceptions highlight the need for works that highlight valid character of historical approaches about physics
\end{abstract}


content. Therefore, considering the importance of HS, we highlight the importance of examining the presence of this relationship in the TB of Physics for higher education, investigating how the historical content for the photoelectric effect is stated in these materials. For this, we build on two criteria: the first refers to the fidelity of the analyzed material compared to the original work, taking into account the historical approach of the photoelectric effect and the second criterion is the analysis of the presence of misconceptions in teaching materials in higher education on historical aspects of science, such as myths, inadequacies and anachronisms.

Keywords: Techno-scientific culture; Dignity of sense; Participatory teaching; Culture of peace.

\section{Aportaciones teóricas y enfoques históricos del Efecto Fotoeléctrico}

\section{Resumen:}

Aumento de la producción de documentos académicos han analizado la presencia de un enfoque histórico en las disciplinas de contenido de la física. Sin embargo, la mayor parte de los resultados de estos estudios no llegan a los profesores, lo que les deja a merced de historias (o historias) distorsionados y/o simplificado, presente en la mayoría de los libros de texto (LDs). Al considerar la posibilidad de utilizar la Historia de la Ciencia (HC) en la educación y la importancia de la enseñanza de un contenido relacionado con la llamada física moderna, es importante examinar cómo es esta articulación en los libros de texto, ya que estos son la principal herramienta de enseñanza que el profesor tiene acceso. El pseudo-historia se encuentra en algunos Física LDs reforzar conocido mito científico y transmitir conceptos históricos falsos a los estudiantes y profesores. Tales percepciones ponen de relieve la necesidad de obras que ponen de relieve el carácter válido de los enfoques históricos sobre el contenido de la física. Por lo tanto, teniendo en cuenta la importancia de HC, destacamos la importancia de examinar la presencia de esta relación en el LDs Física para la educación superior, la investigación de cómo se muestra el contenido histórico para el efecto fotoeléctrico en estos materiales. Para ello, construimos dos criterios: la primera se refiere a la fidelidad del material analizado, en comparación con la obra original, teniendo en cuenta el enfoque histórico del efecto fotoeléctrico y el segundo criterio es el análisis de la presencia de conceptos erróneos en los materiales de enseñanza la educación superior en los aspectos históricos de la ciencia, tales como mitos, insuficiencias y anacronismos.

Palabras clave: Efecto fotoeléctrico; Historia de la Ciencia; Libros de texto; Física Moderna.

\section{INTRODUÇÃO}

Ao considerarmos a possibilidade do uso da História da Ciência (HC) no ensino e a importância de se ensinar um conteúdo relacionado a uma Física dita moderna, torna-se relevante examinar como se dá essa articulação nos livros didáticos, uma vez que esses são a principal ferramenta didática a que o docente tem acesso. A razão de se trabalhar essa temática está diretamente relacionada a situações vivenciadas ao longo da nossa vida estudantil. Durante a nossa formação em Licenciatura em Física bem como no Mestrado, tivemos a oportunidade de discutir alguns fundamentos históricos relativos à Mecânica Clássica, Física Moderna e Quântica, os quais nos forneceram uma visão histórica diferente daquela que tínhamos anteriormente. No desenvolvimento da nossa pesquisa, várias referências bibliográficas (KUHN, 1998; MARTINS, 2005; MARTINS, 2007; MATTHEWS, 1994; 
WHITAKER, 1979) contribuíram para enxergarmos como a abordagem conceitual da HC é trabalhada de maneira estéril, distorcida e superficial.

Nesse contexto, nossa preocupação se volta para os conceitos abordados em materiais didáticos usados na formação inicial de futuros professores de Física, na maioria das Universidades. Como discutido em Martins (2006) e Whitaker (1979), esses materiais estão permeados por inadequações, mediante narrativas que se estruturam em diversos artifícios retóricos, estabelecendo o mito de que servem para explicar e justificar a autoridade da ciência. Segundo Allchin (2003), esses elementos conspiram juntos para desmoronarem a natureza científica em uma pseudo-história, uma vez que se tornam similares ao método como a ciência encontra a verdade.

Assim, diante desse quadro, propusemos o seguinte questionamento: Como a história do efeito fotoelétrico é descrita nos livros de Física (ou Física Moderna) para o ensino superior? Buscando responder a essa questão, inicialmente, recorremos à literatura (KLASSEN, 2009; LEITE, 2002; PAGLIARINI, 2007; NIAZ et al., 2010) que aponta para lacunas e inadequações presentes em conteúdos históricos inclusos nos livros didáticos de Física, no que se refere aos ensinamentos do efeito fotoelétrico. Esses autores apresentam um quadro geral de livros voltados para o nível médio e alguns materiais de divulgação da ciência. Contudo, apenas Niaz et al.(2010) pesquisa sobre livros para a graduação, a partir de uma análise, ainda, bastante superficial. Desse modo, nossa pesquisa visa delinear, de maneira um pouco mais aprofundada, a história do efeito fotoelétrico presente nesses materiais.

\section{METODOLOGIA}

Para respondermos ao questionamento elencado para este estudo - Como a história do efeito fotoelétrico é descrita nos livros de Física (ou Física Moderna) para o ensino superior?verificamos como os autores das obras, selecionadas para análise, exploram e explicam a evolução do fenômeno referente ao efeito fotoelétrico, dentro de um processo de produção cultural e do conhecimento, evitando resumi-la a biografias de cientistas ou a descobertas isoladas. Como corpus de análise selecionamos os seguintes livros: Livro 1: Física moderna (1999); Livro 2: Fundamentos da Física (1999); Livro 3: Física IV: Ótica e física moderna (2009) e Livro 4: Ótica, relatividade e Física quântica: Curso de Física básica (1998), conforme quadro ilustrativo na página seguinte: 
Quadro 1: Referências dos livros analisados

\begin{tabular}{|l|c|}
\hline Livro 1 & $\begin{array}{r}\text { TYPLER, Paul A.; LLEWELLYN, Ralph A.. Física moderna. 3. ed. Rio de Janeiro: LTC, } \\
1999\end{array}$ \\
\hline Livro 2 & $\begin{array}{r}\text { HALLIDAY, David; RESNICK, Robert; WALKER, Jearl. Fundamentos da Física. v.. 4. } \\
\text { 8. ed. Rio de Janeiro: LTC, 2009. }\end{array}$ \\
\hline Livro 3 & $\begin{array}{r}\text { YOUNG, Hugh D.; FREEDMAN, Roger A.. Física IV: Ótica e física moderna. v. 4. 12. } \\
\text { ed. Rio de Janeiro: LTC, 2009. (Título original: Sears and Zemansky). }\end{array}$ \\
\hline Livro 4 & NUSSENZVEIG, Herch Moysés. Ótica, relatividade e física quântica. Curso de \\
Física básica. São Paulo: Blucher, 1998.
\end{tabular}

Fonte: Própria.

Tendo em vista a infinidade de possibilidades que poderiam ser estudadas, é perceptível a necessidade de delimitar nossa pesquisa, norteando-nos para um estudo mais específico. Muitas teorias e muitos experimentos foram realizados para elucidação do efeito fotoelétrico, entretanto não seria viável abordar aqui todos esses episódios, por isso elencamos apenas alguns tópicos. Por conseguinte, elaboramos dois critérios gerais que nortearão nossa pesquisa:

\section{Critério 1: Contribuições teóricas sobre a abordagem histórica do efeito fotoelétrico}

\section{a) Hertz:}

A partir da leitura do artigo escrito por Hertz (1893), pudemos ter maior conhecimento acerca dos trabalhos deste pesquisador, observando que, embora o investigador tenha verificado durante a comprovação das ondas eletromagnéticas o efeito fotoelétrico experimentalmente, ele não se dedicou a esse estudo:

Logo que comecei os experimentos, eu fui afetado por uma notável e recíproca ação entre duas sparks ${ }^{1}$ simultâneas. Eu não tive a intenção de permitir que esse fenômeno distraísse minha atenção do objetivo principal que eu tinha em mente; mas isso ocorreu de um modo tão definido e perplexo que eu não poderia completamente negligenciá-lo (HERTZ, 1893, p. 4).

Assim, embora tenhamos consciência de que esse pesquisador observou experimentalmente o fenômeno, fornecendo importantes contribuições para o seu estudo, a ${ }^{1}$ Centelhas/ faíscas emitidas durante o experimento. (Tradução nossa) 
exemplo da facilitação de emissão de centelhas quando a esfera eletrizada negativamente era iluminada com luz ultravioleta, percebemos que ele estudou suscintamente o fenômeno, não se desviando de seu objetivo (comprovar experimentalmente as equações de Maxwell). Em outra citação, Hertz (1886) reforça que:

Assim que eu soube que estava lidando com um efeito da luz ultravioleta, eu coloquei de lado essa investigação, para dirigir minha atenção para a questão principal mais uma vez. Entretanto, como certa familiaridade com o fenômeno é requerida na investigação das oscilações, eu publiquei um comunicado relatando esse fato (HERTZ, 1886, apud MANGILI, 2012, p.46).

No entanto, fica aqui um questionamento: será que Hertz sabia que tinha descoberto o efeito fotoelétrico? Essa é uma questão que levanta algumas discussões, uma vez que, historicamente, existem alguns critérios que necessitam serem atendidos, para que se possa realizar uma descoberta. Nesse sentido, tomando como base o artigo de Martins (1999), que relata sobre uma série de critérios que devem ser atendidos para que se possa caracterizar a descoberta de um novo fenômeno, podemos dizer que é inconveniente atribuir a ele Hertz essa descoberta. A partir dessas informações, buscamos verificar como esse episódio histórico é abordado nos livros aqui analisados.

\section{b) Lenard:}

Por meio da leitura dos artigos escritos por Lenard (1902; 1906), podemos afirmar que ele forneceu importantes contribuições para a elucidação do efeito fotoelétrico, ao discutir sobre a hipótese do gatilho, determinando que a velocidade dos fotoelétrons ejetados independa da intensidade da luz e que a intensidade da corrente desses fotoelétrons aumente com a frequência. A ideia de Philipp Lenard foi amplamente aceita e considerada a sugestão mais correta para a explicação do fenômeno até aproximadamente 1910, como aponta Wheaton (1983). Segundo Niaz et al., (2010), a hipótese do gatilho teria sido uma das maiores contribuições na elucidação do fenômeno. Por isso, é importante que esse teórico seja contemplado nos livros. Perante essas considerações, nosso objetivo consiste na verificação da presença desse conteúdo nas obras analisadas e na maneira como ele é apresentado. 


\section{c) Einstein}

As contribuições apresentadas por Albert Einstein em 1905 para elucidação do efeito fotoelétrico, escrevendo uma nova teoria para descrever o fenômeno e sumarizando a famosa equação fotoelétrica, foi por ele próprio considerado revolucionário, pois, além de se tratar de um trabalho heurístico, estava em desacordo com as teorias vigentes na época (teoria ondulatória). Utilizando a hipótese quântica, Einstein conseguiu explicar, de forma relativamente simples, o fenômeno em questão, quebrando um paradigma defendido por Planck e recorrendo à lei de W. Wien para fundamentar seu trabalho. Einstein mostrou que a expressão para dependência volumétrica da entropia da radiação, dentro dos limites de uma determinada frequência, tem forma similar à entropia de um gás ideal, concluindo que a radiação monocromática de baixa densidade se comporta, termodinamicamente, como sendo constituída de quantum de energia de magnitude Apesar da analogia dessa expressão com a constante de Planck (h), a ideia de Einstein foi explorar uma analogia que descobriu entre as expressões da entropia para a radiação emitida por um corpo negro (não dos osciladores como pensou Planck), no limite de validade da lei de Wien, e da entropia de um gás dada pela teoria cinética (JAMMER, 1966). Nesse sentido, verificamos como o trabalho de Einstein para o efeito fotoelétrico é exposto nos livros analisados.

\section{d) Millikan}

Após a publicação do trabalho de Einstein em 1905, vários pesquisadores começaram a desenvolver, individualmente, pesquisas para verificarem, experimentalmente, a teoria de Einstein. Boa parte das pesquisas desenvolvidas buscava demonstrar que a teoria einsteiniana estava equivocada, como assinala Wheaton (1983). A maioria desses estudiosos não obteve aceitação em suas teorias, visto que não conseguiam explicar e nem reproduzir o fenômeno com clareza. A explicação para o fenômeno só foi aceita no ano de 1916, com os trabalhos realizados por Robert Millikan, que se destacou como físico experimental e pela qualidade dos seus resultados em comparação aos físicos anteriores. Millikan (1916) tinha o intuito de comprovar, experimentalmente, que Einstein (1905) estava equivocado, no entanto, seu trabalho evidencia a validade geral da equação de Einstein, mesmo que continuasse discordando de sua teoria. Nesse último ponto a ser avaliado, dedicamo-nos a verificar como os livros didáticos apresentam o conteúdo referente às contribuições de Millikan (1916) para o estudo do efeito fotoelétrico. 


\section{Critério 2: A presença de concepções inadequadas em materiais didáticos do ensino} superior sobre aspectos históricos da ciência, como mitos, inadequações e anacronismos

Verificamos a possível presença de situações inadequadas na descrição de um episódio histórico presentes nesses materiais. Desse modo, analisamos se os livros apresentam: descrição do fenômeno pela simples sucessão de datas e nomes, bem como a ideia de uma ciência linear, desenvolvida por grandes gênios e imune ao erro; ademais, se há presença de anacronismos, pseudo-histórias e mitos.

\section{ANÁLISE E DISCUSSÃo DOS RESULTADOS}

No que se refere ao critério 1, Contribuições teóricas sobre a abordagem histórica do efeito fotoelétrico, apresentado no tópico anterior, observamos que todos os livros analisados apresentam, em determinado momento, algum relato histórico sobre esse episódio.

Os livros 1, 3 e 4 discutem sobre a primeira verificação para o efeito fotoelétrico, que teria sido realizada por Hertz (1983), mas não atribui a ele essa descoberta, o que pode ser considerado bastante positivo, como pode ser observado na seguinte colocação: "O efeito fotoelétrico foi observado inicialmente por Heinrich Hertz em 1887 (...)" (YOUNG; FREEDMAN, 2009, p.181. Grifo nosso). O livro 2 já não discute sobre as contribuições desse autor para a elucidação do fenômeno, utilizando os aspectos históricos apenas para trazer uma demonstração rústica do aparato experimental desenvolvido por Hertz, para o estudo das ondas eletromagnéticas.

Os livros 1, 3 e 4 discutem sobre as contribuições dadas por Lenard (1902), com a hipótese do gatilho, porém não utilizam essa nomenclatura. O livro 1, por exemplo, argumenta que "ao contrário do que previa a teoria clássica, não foi observada uma intensidade mínima abaixo da qual a corrente fosse nula. (...) o potencial não depende da intensidade da luz incidente" (TIPLER; LLEWELLYN, 2009, p. 88-89).

Quando se discute o trabalho de Lenard (1902), a argumentação sobre a incompatibilidade do efeito fotoelétrico com a Física Clássica aparece frequentemente nesses livros (1, 3 e 4). Isso pode ser percebido na citação anterior e ainda na seguinte 
argumentação: “As investigações posteriores do efeito fotoelétrico, devidas principalmente a P. Lenard (1899), revelam uma série de características intrigante, contraditórias ao que seria esperado pela física clássica" (NUSSENZVEIG, 1998, p. 249). Nessa citação, Nussenzveig (1998) parece corroborar com o fato de que Lenard foi muito importante ao revelar fatos, mas falhou ao explica-los.

No que se refere ao trabalho desenvolvido por Einstein, o livro 1 coloca que para Einstein "a luz é constituída por quanta isolados de energia hf. Quando um desses quanta, denominados fótons, chega à superfície do catodo, toda sua energia é transferida para um elétron" (TIPLER; LLEWELLYN, 2009, p. 89). De acordo com o livro 3, "Einstein postulou que um feixe de luz era constituído por pequenos pacotes de energia, chamados fótons ou quanta" (YOUNG; FREEDMAN, 2009, p. 183). No livro 4, a argumentação de Einstein nos diz que "A ideia mais simples é que um quantum de luz transfere toda sua energia para um único elétron. (...) 0 nome fóton para um quantum de luz só aparece em 1926 num trabalho de G.N. Lewis" (NUSSENZVEIG, 1998, p. 252-253). Como podemos perceber, salvo o livro 4, a maioria associa o termo fóton à descrição do fenômeno proposto por Einstein. Essa associação é equivocada, uma vez que ele não utilizou este termo durante a descrição do fenômeno.

O livro 1 comenta sobre a verificação experimental de Millikan (1916), mostrando que o valor obtido nesse experimento é muito próximo ao valor definido para a constante de Planck e traz um gráfico (simplificado) dos resultados obtidos por ele, conforme podemos comprovar na figura 1:

Figura 1: Dados obtidos por Millikan para o potencial de corte em função da frequência

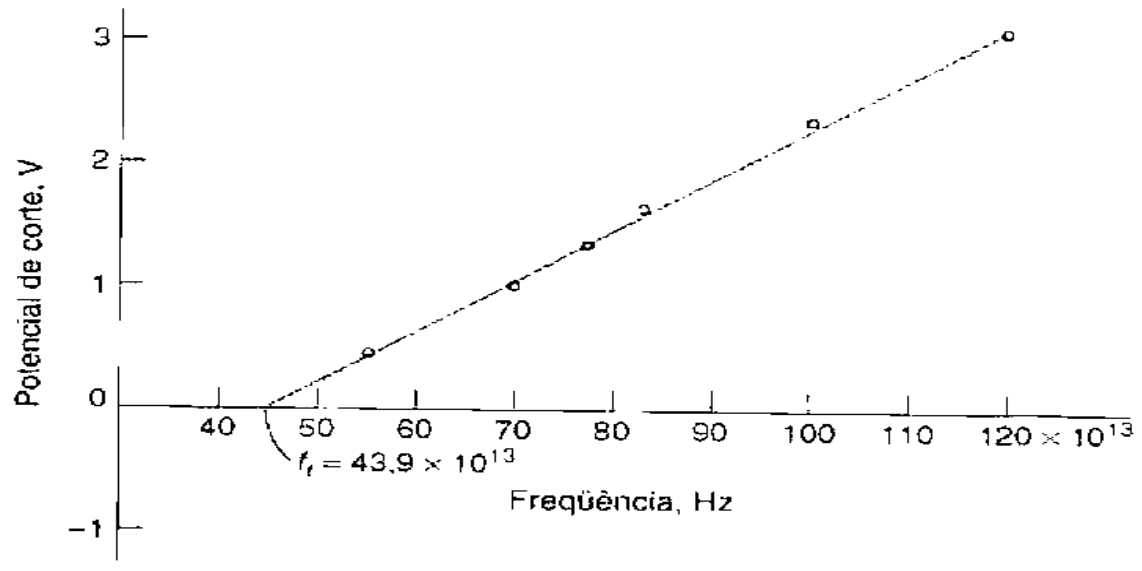

Fonte: TIPLER; LLEWELLYN, 2009, p. 89. 
Em linhas gerais, o livro 1 discute o episódio histórico de forma precisa, não cometendo equívocos sobre a sua descrição. Um ponto bastante positivo desse livro deve-se ao fato de os autores recorrerem, constantemente, a materiais originais para fundamentarem suas argumentações. No livro 2, a argumentação histórica é utilizada apenas para descrição experimental do fenômeno. Os autores colocam uma figura, aparentemente atual, para ilustrar o aparato experimental (Fig. 2), mas não apresentam nenhuma explicação sobre ele. Não há descrições sobre o aparato experimental, bem como as variáveis e os procedimentos realizados. São utilizados termos estranhos como: "contato deslizante", “coletor", "ligeiramente negativo", "alvo", entre outros, o que acaba dificultando o entendimento, porque não são termos usuais e não há nenhuma descrição sobre eles, de acordo o transcrito na figura a seguir (figura 2):

Figura 2: Esquema utilizado para descrever experimentalmente o efeito fotoelétrico

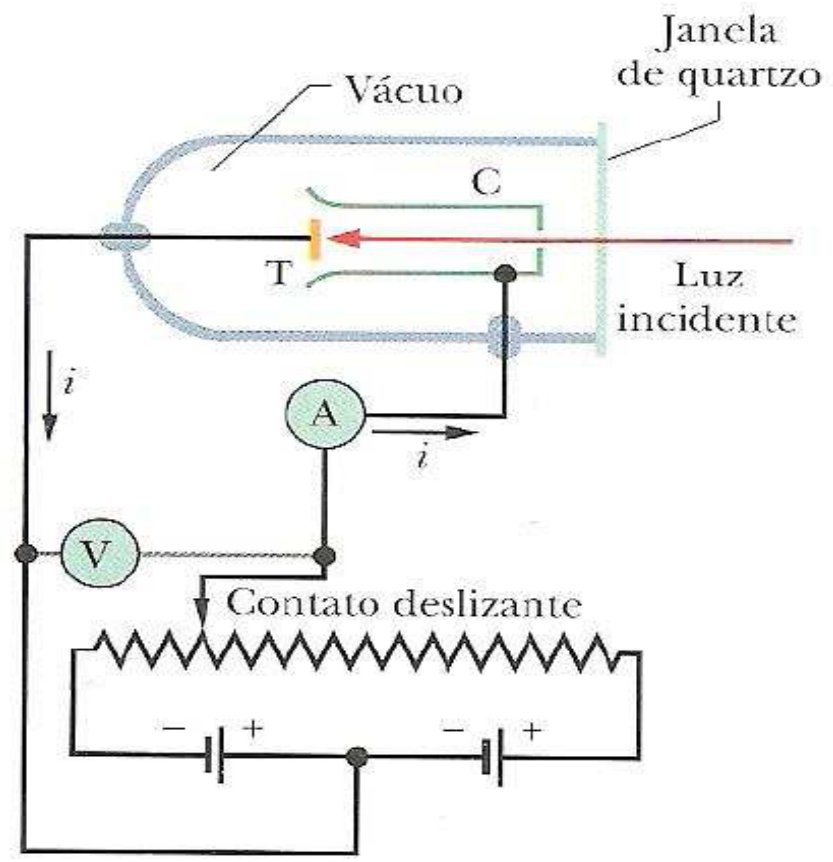

Fonte: HALLIDAY, RESNICK, WALKER, 1999, p.188.

O livro 3 apresenta o conteúdo histórico de forma bem interessante, porquanto consegue fazer uma ligação entre o estudo que fazemos na atualidade e a descrição histórica do efeito fotoelétrico. Embora seus autores cometam alguns equívocos de nomenclatura, pois utilizam termos não empregados nos originais, o conteúdo é, em sua maioria, conceitual e atual, mesmo respeitando o desenvolvimento histórico. 
O livro 4 traz a história do efeito fotoelétrico apresentada de forma mais precisa. É notável a preocupação do autor em descrever o episódio, respeitando o desenvolvimento dos fatos. Mesmo ao utilizar a constante de Planck e assimilar esse procedimento àquele desenvolvido por Einstein, esse pequeno equívoco não compromete a qualidade do material apresentado.

Quanto ao segundo critério A presença de concepções inadequadas em materiais didáticos do ensino superior sobre aspectos históricos da ciência, como mitos, inadequações e anacronismos, de uma maneira geral, podemos colocar que ainda existem algumas lacunas e inadequações nessa tentativa de estabelecer explicações mais eficiente. Os problemas enfrentados para estabelecer uma nova hipótese não são mostrados, assim como as incongruências dentro das próprias teorias. A evolução histórica é apresentada por um percurso que não ocorre na prática, além de ser marcada excessivamente por datas e nomes, fazendo-nos crer em sua linearidade. Isso pode ser percebido na seguinte argumentação: “A análise correta do efeito fotoelétrico foi feita por Albert Einstein em 1905" (YOUNG; FREEDMAN, 2009, p.183).

Constantemente, os autores recorrem a anacronismos para explicar os fatos, como, por exemplo, a utilização de constantes, nomenclatura e conceitos que não fazem parte do trabalho desenvolvido na época.

\begin{abstract}
Einstein postulou que um feixe de luz era constituído por pequenos pacotes de energia, chamados fóton ou quanta. A energia $E$ do fóton é igual a uma constante $h$ vezes a frequência. (...) Um fóton que atinge uma superfície é absorvido por um elétron. Essa transferência é do tipo tudo ou nada, ou seja, o elétron ou ganha a energia total do fóton ou não absorve nenhuma energia (...) (YOUNG; FREEDMAN, 2009, p. 183. Grifo nosso).
\end{abstract}

Mesmo que esse seja um recurso utilizado para fins pedagógicos, esse anacronismo acaba por danificar o conhecimento, ao passar ideias equivocadas para os alunos. Como sugestão, defendemos que ao utilizar anacronismos, esses sejam explicados no processo de ensino-aprendizagem, por exemplo, através de notas de rodapé ou barras laterais nos livros. 


\section{CONCLUSÃO}

Ao analisarmos, nesse artigo, recorte de um estudo mais amplo referente à dissertação de mestrado, o contexto histórico sugerido em nossa pesquisa e posto em alguns dos livros, selecionados para análise, pudemos constatar que, na maioria deles, há uma inconsistência e superficialidade nos argumentos apresentados, levando-nos a verificar que características como a excessiva simplificação, matematização e falta de contexto fornecem informações inadequadas e desatualizadas sobre o efeito fotoelétrico. Destarte, esses resultados, segundo alguns autores previamente consultados, já haviam sido demonstrados em outras pesquisas.

Embora haja, em alguns livros didáticos aqui analisados, um estímulo explícito à leitura desse fenômeno e as informações apresentadas sejam corretas do ponto de vista histórico, esses aspectos são pouco explorados ou quando são é de maneira superficial. Temos consciência de que nossa pesquisa não consegue abranger todos os pontos relevantes referentes ao efeito fotoelétrico e ainda existem muitas lacunas a serem preenchidas, todavia, ressaltamos que esse trabalho não é apresentado como uma pesquisa concluída e imutável. Esse aprofundamento é ponto ao qual nos deteremos em pesquisas futuras.

\section{REFERÊNCIAS}

ALLCHIN, D.. Scientific myth-conceptions. Science Education, v. 87, n. 3, p. 329-351, 2003.

EINSTEIN, A.. On a heuristic point of view concerning the production and transformation of light. Annalen der Physik, vol. 17, p. 132-148, 1905. Disponível em < http://www.einsteinpapers.press.princeton.edu/vol2trans $>$. Acesso em 20 de outubro de 2014.

HALLIDAY, D.; RESNICK, R.; WALKER, J.. Fundamentos da Física. v.. 4. 8. ed. Rio de Janeiro: LTC, 2009.

HERTZ, H. R.. Electric waves: Being Researches on the propagation of electric Action With Finite Velocity Through Space. London; Macmillan and Co, 1893.

KLASSEN, S.. The photoelectric effect: Reconstructing the story for the physics classroom. Science \& Education, v.18, p. 593-607, 2009.

KUHN, T.. A Estrutura das Revoluções Científicas. [1962]. Ed Perspectiva, São Paulo, (1998).

LEITE, L.. History of science in science education: Development and validation of a checklist for analyzing the historical content of science textbooks. Science \& Education, v. 11, n. 4, p. 333-359, 2002.

LENARD.P.E.A.V.. "Uber die lichtelektrische Wirkung." AP, 5 Leipzig, (1902), 149-198. 
On cathode rays. Nobel lecture, may 28, 1906. p.105-134

MANGILI, A. I.. Heinrich Rudolph Hertz e a descoberta do efeito fotoelétrico: Um exemplo dos cuidados que devemos ter ao utilizar a história da ciência na sala de aula. História da ciência e ensino: construindo interfaces. v.6, p. 32-48, 2012.

MARTINS, A.F.P.. História e filosofia da ciência no ensino: há muitas pedras nesse caminho... Caderno Brasileiro de Ensino de Física, Florianópolis, v. 24, n. 1, p. 112-131, 2007.

MARTINS, L A-C. P.. História da ciência: objetos, métodos e problemas. Ciência \& Educação, v. 11, n. 2, p. 305317, 2005.

MARTINS, R. A.. A maçã de Newton: História, Lendas e Tolices. In: SILVA, C. C. Estudos de história e filosofia das ciências: subsídios para aplicação no ensino. São Paulo: Editora Livraria da Física, 2006.

- ¿Que es descubrimiento cientifico de um nuevo fenómeno? In: SOTA, Eduardo \& URTUBEY, Luis (eds.) Epistemología e Historia de la Ciência. Córdobra: Universidad Nacional de Córdobra, 1999. Vol. 5 (n.5), pp. 281-288.

MATTHEWS, M. R.. Science Teaching - The Role of History and Philosophy of Science. New York: Routledge, 1994.

MILLIKAN, R. A.. “A Direct Photoelectric Determination of Planck's “h”, Physical Review, Second Series, vol. VII, $\mathrm{n}^{\circ} 3$, p. 355-388, (1916).

NÍAZ, M.; KLASSEN, S.; MC MILLAN, B.; METZ, B.. Reconstruction of the history of the photoelectric effect and its implications for general physics textbooks, Science Education, 94, 903-931, 2010.

NUSSENZVEIG, H. M.. Ótica, relatividade e física quântica. Curso de Física básica. São Paulo: Blucher, 1998.

PAGLIARINI, C.R.. Uma análise da história e filosofia da ciência presente em livros didáticos de Física para o ensino médio. 115f. Dissertação (Mestrado em Ciências) - Instituto de Física de São Carlos, Universidade de São Paulo, São Carlos, 2007. Disponível em <http://www.educadores.diaadia.pr.gov.br/arquivos/File/2010/artigos teses/Ciencias/Diss rtacoes/1017.pdf>. Acesso em 15 de abril de 2016.

TYPLER, P. A.; LLEWELLYN, R. A.. Física moderna. 3. ed. Rio de Janeiro: LTC, 1999

YOUNG, H. D.; FREEDMAN, R. A.. Física IV: Ótica e física moderna. v. 4. 12. ed. Rio de Janeiro: LTC, 2009.

WHEATON, B. R.. Philipp Lenard and the photoelectric effect, 1889 - 1911. Historical Studies in the Physical Sciences, 9, 199 - 322. (1983).

WHITAKER, M. A. B.. History and quasi-history in physics education. Part I, Physics education, vol. 14, p. 108 112, 1979. 